\title{
SIGNIFICANCE OF RADIOLOGICAL CALCIFICATION OF JOINT CARTILAGE
}

\author{
BY \\ H. L. F. CURREY, J. J. KEY, R. M. MASON, AND K. V. SWETTENHAM \\ The London Hospital, Whitechapel, London
}

Sporadic reports of radiological calcification of knee menisci have appeared in the literature for many years, but it was not until recently that Žitňan and Sitaj $(1958,1960,1963)$ established the association of joint cartilage calcification and arthropathy. These authors described the familial occurrence of acute and chronic arthritis in patients with calcification of cartilage in multiple joints. A similar radiological appearance was then observed by McCarty, Kohn, and Faires (1962) in patients with attacks of acute arthritis attributed to the presence of crystalline calcium pyrophosphate within affected joints. It now appears that there is at least considerable overlap between the "chondrocalcinosis" of Žitňan and Sitaj, and the "pseudo-gout" of McCarty, Kohn, and Faires. A similar pattern of joint calcification may be present in patients with functioning parathyroid adenomata (Bywaters, Dixon, and Scott, 1963); such patients may also develop joint disease (Brit. med. J., 1964).

A reappraisal of the significance of joint cartilage calcification would therefore appear to be desirable. Increasing awareness has shown that this is a not uncommon radiological finding, but its interpretation is often uncertain. This is particularly so when calcification is inconspicuous and limited, for example, to one knee meniscus.

To evaluate the diagnostic significance of this finding in clinical practice, we have investigated all patients encountered over the past 2 years with radiological calcification in at least one joint.

\section{Material and Methods}

A total of 35 cases was found; 27 were patients attending the London Hospital, seven were referred from other hospitals, and one was a medical colleague. Each was questioned and examined by one of the authors. Synovial fluid was obtained for examination on at least one occasion from 23 of them. Two drops of the fluid were placed on a glass slide, covered by a coverslip, and sealed with vaseline. Polarized light microscopy was used to search for and identify the optical properties of the crystals. Phase contrast microscopy was useful for observing morphology, but was not used routinely. Laboratory investigations included determinations of serum values for calcium, inorganic phosphorus, alkaline phosphatase, urea, and uric acid. Material for histological examination was obtained at operation upon the joints of five patients.

\section{Results}

Clinical Features.-All 35 patients had at some time signs and symptoms of joint disease. There were 21 females and 14 males. Their average age when seen was 64 years (range 32-89) and at the onset of the first joint symptoms 52 years (range 30-74). For purposes of description the 35 cases have been subdivided into five groups (Table I).

TABLE I

CLINICAL PATTERNS OF JOINT DISEASE IN 35 PATIENTS WITH RADIOLOGICAL CALCIFICATION OF JOINT CARTILAGE

\begin{tabular}{|c|c|c|c|}
\hline 1 & \multicolumn{2}{|c|}{$\begin{array}{l}\text { Episode(s) of acute arthritis in one or a few large } \\
\text { joints, some going on to develop osteo-arthrosis }\end{array}$} & 13 \\
\hline 2 & $\begin{array}{l}\text { Osteo-arthrosis of few } \\
\text { or many joints }\end{array}$ & $\begin{array}{l}\text { (a) With acute episodes } \\
\text { (b) Without acute } \\
\text { episodes }\end{array}$ & $\begin{array}{l}7 \\
7\end{array}$ \\
\hline 3 & \multicolumn{2}{|c|}{ Polyarthritis mimicking rheumatoid arthritis } & 3 \\
\hline 4 & Classical uric acid gout & $\begin{array}{l}\text { (a) Acute episodic arthri- } \\
\text { tis } \\
\text { (b) Episodes of acute } \\
\text { podagra, then sym- } \\
\text { metrical polyarthritis } \\
\text { resembling rheuma- } \\
\text { toid arthritis }\end{array}$ & 2 \\
\hline 5 & Hyperparathyroidism & $\begin{array}{l}\text { (a) Acute arthritis of one } \\
\text { knee joint } \\
\text { (b) Nodal multiple osteo- } \\
\text { arthrosis }\end{array}$ & $\begin{array}{l}1 \\
1\end{array}$ \\
\hline & \multicolumn{2}{|l|}{ Total } & 35 \\
\hline
\end{tabular}

Group 1.-Thirteen patients had one or more attacks of acute arthritis with effusion affecting large joints. These tended to involve one or a few joints at a time and subsequent attacks often returned to the same joint. Knees 
were much the commonest site. The clinical features were often acute enough to suggest septic arthritis, and two patients (Cases 5 and 12) were actually treated as such (Hamblen, Currey, and Key, 1966). During attacks constitutional disturbances were present with malaise and fever, but leucocytosis was unusual. Patients who had experienced attacks over a number of years usually showed some evidence of osteo-arthrosis in the affected joints and, to a lesser extent, elsewhere.

Group 2.-Fourteen patients presented a picture of generalized osteo-arthrosis. Apart from severity this in no way differed from the typical pattern of degenerative joint disease, with the emphasis on weight-bearing joints and, in four cases, Heberden's nodes and arthrosis of the first carpo-metacarpal joint. Half of these osteoarthritic cases (seven) gave a history of acute or subacute attacks of arthritis in affected joints. These attacks were milder than those described by Group 1 patients, and occurred only against a background of established osteoarthrosis. The other seven had no acute episodes.

Group 3.- Three patients presented a clinical picture mimicking rheumatoid arthritis. Each had a symmetrical polyarthritis affecting many small and large joints, with considerable constitutional disturbance. In two this occurred at very infrequent intervals over many years, each attack lasting a few weeks. In the third patient a similar picture had evolved gradually over 16 years. All three patients had been diagnosed as suffering from rheumatoid arthritis, but none had nodules or definite radiological erosions, and tests for rheumatoid factor were negative. In two, synovial histology showed no rheumatoid features.

Group 4.-Three patients were thought to be suffering from classical uric acid gout. Of these, one (Case 31) had recurrent episodes of acute arthritis including podagra, hyperuricaemia, and urate crystals in his synovial fluid. One (Case 32) had only a single attack of arthritis, affecting a knee and a 5th metacarpophalangeal joint. No synovial fluid was available for examination, but he was hyperuricaemic and had a strong family history of gout.

The third patient (Case 33) had a 4-year history of episodes of acute podagra and then developed a severe symmetrical small and large joint polyarthritis mimicking rheumatoid arthritis (erythrocyte sedimentation rate $130 \mathrm{~mm}$./1st hour). She had neither rheumatoid nodules nor radiological erosions, and tests for rheumatoid factor were negative. Plasma uric acid levels were consistently elevated, and she appeared to respond to colchicine. Her synovial fluid contained a mixture of urate, and crystals of what was thought to be calcium oxalate.

Group 5.-Two patients had hyperparathyroidism. One (Case 34) had a functioning parathyroid adenoma resected and 2 years later experienced a single attack of acute arthritis in one knee. The other (Case 35) presented with what appeared to be nodal multiple osteoarthrosis. Her serum calcium level was elevated and further investigations established hyperparathyroidism. She was considered unsuitable for surgery.

Radiology.-The radiological distribution of joint calcification is shown in Table II (opposite). The radiology, based on the first fifteen patients in this series, has been described in detail elsewhere(Key and Currey, in press); the remaining cases showed similar general features. Examples of the typical appearances are illustrated in Figs 1 to 6 (overleaf).

The distribution of joint calcification among all 35 patients is shown in Table II. There was no tendency for calcification to be more marked in older patients. No difference in extent or distribution of calcification was apparent between the various diagnostic groups.

History.-Few patients gave a history of significant joint disease in relatives. Table III (overleaf) indicates those who had a positive family history, and also lists the associated diseases found in these patients.

Laboratory Investigations.-The results of some relevant biochemical investigations are included in Table III. There were five patients (Cases 5, 16, 17, 19 , and 23 ) in whom no evidence of bone or liver disease could be found to account for a raised serum level of alkaline phosphatase. Similarly, a number of patients thought not to be suffering from classical gout had raised serum uric acid levels. Whilst it is not possible to exclude, for certain, that some of these might have been produced by therapy, specific inquiry was made into this point. In in-patients, aspirin or other relevant drugs were excluded. In out-patients, however, it was not possible to be sure that the patient was not taking small doses of aspirin.

Synovial Fluid Examination.-The general properties of the fluids examined were similar to those described by McCarty, Kohn, and Faires (1962). Fluids from acutely inflamed joints were opaque, with a high cell content and low viscosity. One was markedly haemorrhagic. Fluids from quiescent joints had fewer cells and normal viscosity. Crystals o were present in all but two specimens, although often in very small numbers.

Thirteen fluids contained crystals with the optical properties of calcium pyrophosphate (McCarty, Kohn, and Faires, 1962). Needle and rod forms were seen both within cells and lying free, while $\stackrel{\Phi}{\Phi}$ rhomboid forms were usually extracellular. They varied in length from 2 to 15 microns. All exhibited weak positive birefringence. Three fluids contained typical urate crystals-needles showing strong 
TABLE II

DISTRIBUTION OF CALCIFICATION IN JOINT CARTILAGE

Under each heading the first column represents the joint on the right side, and the second column the joint on the left side

\begin{tabular}{|c|c|c|c|c|c|c|c|c|c|c|c|}
\hline Group & $\begin{array}{l}\text { Case } \\
\text { No. }\end{array}$ & $\begin{array}{l}\text { Medial } \\
\text { Meniscus }\end{array}$ & $\begin{array}{c}\text { Lateral } \\
\text { Meniscus }\end{array}$ & Pubis & Wrist & Knee & Shoulder & Hip & Elbow & Ankle & $\begin{array}{c}\text { Small } \\
\text { Joints } \\
\text { of Hands } \\
\text { and Feet }\end{array}$ \\
\hline \multirow{13}{*}{1} & 1 & $-\quad+$ & $+\quad+$ & - & $-\quad-$ & $-\quad+$ & & & & & $-\quad-$ \\
\hline & 2 & $-\quad+$ & $-\quad+$ & - & $-\quad-$ & $-\quad-$ & $-\quad-$ & $-\quad-$ & $-\quad-$ & -- & $-\quad-$ \\
\hline & 3 & $+\quad+$ & $+\quad+$ & + & $+\quad+$ & ++ & $+\quad-$ & $+\quad+$ & & $-\quad+$ & $-\quad-$ \\
\hline & 4 & $+\quad+$ & $+\quad+$ & - & $-\quad-$ & ++ & $+\quad+$ & +- & $+\quad+$ & $-\quad-$ & $-\quad-$ \\
\hline & 5 & $+\quad+$ & $+\quad+$ & + & ++ & ++ & $+\quad+$ & $+\quad+$ & $+\quad+$ & ++ & $-\quad-$ \\
\hline & 6 & ++ & $+\quad+$ & - & $-\quad-$ & -- & -+ & $-\quad-$ & $+\quad+$ & $-\quad-$ & $-\quad-$ \\
\hline & 7 & $+\quad+$ & $+\quad+$ & - & $-\quad-$ & $-\quad-$ & & $-\quad-$ & - & - & $-\quad-$ \\
\hline & 8 & $+\quad+$ & $+\quad+$ & + & $-\quad+$ & ++ & $+\quad+$ & ++ & $+\quad+$ & & $-\quad-$ \\
\hline & 9 & $-\quad+$ & $-\quad-$ & + & $-\quad-$ & $-\quad-$ & $-\quad-$ & $-\quad-$ & $-\quad-$ & & $-\quad-$ \\
\hline & 10 & $+\quad+$ & $+\quad+$ & + & $-\quad-$ & $-\quad-$ & $-\quad-$ & $-\quad-$ & 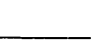 & & $-\quad-$ \\
\hline & 11 & $+\quad+$ & $+\quad+$ & & 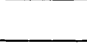 & ++ & $-\quad-$ & 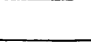 & 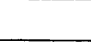 & $-\quad-$ & $-\quad-$ \\
\hline & 12 & $+\quad+$ & ++ & - & $-\quad-$ & $+\quad+$ & $-\quad-$ & $-\quad-$ & -- & & $-\quad-$ \\
\hline & 13 & ++ & $+\quad+$ & + & + & $+\quad+$ & $+\quad+$ & $-\quad-$ & 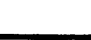 & 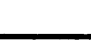 & $-\quad-$ \\
\hline \multirow{14}{*}{2} & 14 & $-\quad+$ & +- & - & $-\quad+$ & $-\quad-$ & $-\quad-$ & $-\quad-$ & $+\quad+$ & 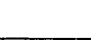 & +- \\
\hline & 15 & $+\quad+$ & ++ & + & ++ & $-\quad-$ & $-\quad-$ & $-\quad-$ & & $-\quad-$ & $-\quad-$ \\
\hline & 16 & $+\quad+$ & +- & + & $-\quad-$ & $-\quad-$ & $-\quad-$ & -- & & - & $-\quad-$ \\
\hline & 17 & $+\quad+$ & $+\quad+$ & + & - & $+\quad+$ & 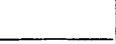 & $-\quad-$ & & & 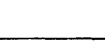 \\
\hline & 18 & $+\quad+$ & $+\quad+$ & - & $-\quad-$ & $+\quad+$ & 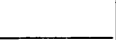 & $-\quad-$ & & & $-\quad-$ \\
\hline & 19 & ++ & $+\quad+$ & - & ++ & ++ & $-\quad-$ & $-\quad-$ & $-\quad-$ & & $-\quad-$ \\
\hline & 20 & $-\quad+$ & $-\quad+$ & - & -+ & $-\quad-$ & $-\quad-$ & -- & & & -- \\
\hline & 21 & $+\quad+$ & $+\quad+$ & - & . & $-\quad-$ & $-\quad-$ & -- & & & $-\quad-$ \\
\hline & 22 & $+\quad+$ & $+\quad+$ & - & $-\quad-$ & -- & $-\quad-$ & $-\quad-$ & & & $-\quad-$ \\
\hline & 23 & $+\quad+$ & ++ & - & $-\quad-$ & +- & $-\quad-$ & $-\quad-$ & & $-\quad-$ & -- \\
\hline & 24 & $+\quad+$ & $+\quad+$ & + & $-\quad-$ & ++ & & & & & \\
\hline & 25 & - & + & & + & - & & & & & \\
\hline & 26 & $+\quad-$ & $+\quad-$ & - & $-\quad-$ & $+\quad-$ & $-\quad-$ & $-\quad-$ & $-\quad-$ & $-\quad-$ & $-\quad-$ \\
\hline & 27 & $+\quad+$ & $+\quad+$ & + & ++ & $+\quad+$ & $+\quad+$ & ++ & ++ & & $+\quad+$ \\
\hline \multirow{3}{*}{3} & 28 & -+ & $-\quad+$ & + & ++ & $-\quad-$ & $-\quad-$ & $-\quad-$ & & - & $-\quad-$ \\
\hline & 29 & $+\quad+$ & $+\quad+$ & - & $-\quad-$ & $+\quad+$ & - & -- & & & $-\quad-$ \\
\hline & 30 & ++ & $+\quad+$ & & ++ & $+\quad+$ & & & ++ & & $+\quad+$ \\
\hline \multirow{3}{*}{4} & 31 & ++ & ++ & - & 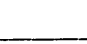 & $+\quad+$ & & $-\quad-$ & & - & $-\quad-$ \\
\hline & 32 & $-\quad+$ & $-\quad+$ & & - & -+ & & & & & $-\quad-$ \\
\hline & 33 & +- & +- & & $-\quad-$ & $-\quad-$ & & & $-\quad-$ & - & $-\quad-$ \\
\hline \multirow{2}{*}{5} & 34 & $+\quad+$ & $+\quad+$ & + & ++ & $-\quad+$ & $+\quad-$ & $+\quad+$ & $-\quad+$ & & $-\quad-$ \\
\hline & 35 & $-\quad-$ & $+\quad+$ & + & $+\quad+$ & $-\quad-$ & $-\quad-$ & $-\quad-$ & $-\quad-$ & $-\quad-$ & $-\quad-$ \\
\hline
\end{tabular}

$+=$ Calcification present. $\quad-=$ Calcification absent. Blank $=$ Not $x$ rayed. 
SUMMARY OF DATA ON 35 PATIENTS

\begin{tabular}{|c|c|c|c|c|c|c|}
\hline Group & $\begin{array}{l}\text { Case } \\
\text { No. }\end{array}$ & Sex & $\begin{array}{c}\text { Age at } \\
\text { Onset } \\
(y r s)\end{array}$ & $\begin{array}{l}\text { Age when } \\
\text { Seen } \\
(y r s)\end{array}$ & $\begin{array}{c}\text { Diagnostic } \\
\text { Category } \\
\text { (see Table I) }\end{array}$ & Other Features including Family History \\
\hline \multirow{13}{*}{1} & 1 & $\mathbf{F}$ & 58 & 61 & 1 & \\
\hline & 2 & $\mathbf{M}$ & 50 & 51 & 1 & จ \\
\hline & 3 & $\mathbf{M}$ & 41 & 54 & 1 & Intestinal malabsorption. Father with "gout" \\
\hline & 4 & $\mathbf{M}$ & 51 & 58 & 1 & \\
\hline & 5 & $\mathbf{M}$ & 55 & 55 & 1 & $\begin{array}{l}\text { Treated as acute septic arthritis } \\
\text { Mild diabetes }\end{array}$ \\
\hline & 6 & $\mathrm{M}$ & 57 & 67 & 1 & Paget's disease left femur. Histology \\
\hline & 7 & $\mathbf{M}$ & 30 & 32 & 1 & $\stackrel{2}{2}$ \\
\hline & 8 & $\mathbf{M}$ & 60 & 69 & 1 & Syringomyelia \\
\hline & 9 & $\mathrm{~F}$ & 48 & 63 & 1 & Diabetes. Hypertension. Cardiac failure \\
\hline & 10 & $\mathbf{F}$ & 48 & 71 & 1 & Carcinoma floor of mouth \\
\hline & 11 & $\mathbf{F}$ & 63 & 63 & 1 & Initial attack 7 th day post-cholecystectomy \\
\hline & 12 & $\mathbf{M}$ & 51 & 60 & 1 & Chronic bronchitis. Treated as acute septic arthritis \\
\hline & 13 & $\mathbf{M}$ & 49 & 61 & 1 & $\subseteq$ \\
\hline \multirow{14}{*}{2} & 14 & $\mathbf{F}$ & 56 & 71 & $2 a$ & Ischaemic heart disease with cardiac failure \\
\hline & 15 & $\mathbf{F}$ & 61 & 62 & $2 a$ & Father with "gout" \\
\hline & 16 & $\mathbf{F}$ & 46 & 59 & $2 a$ & \\
\hline & 17 & $\mathrm{~F}$ & 70 & 70 & $2 a$ & Almost pure blood aspirated from knee. Histology \\
\hline & 18 & $\mathrm{~F}$ & 57 & 62 & $2 a$ & Chronic nephritis. Hypertension. Grandfather with "gous \\
\hline & 19 & $\mathbf{F}$ & 33 & 60 & $2 a$ & Heberden's nodes \\
\hline & 20 & $\mathbf{M}$ & 68 & 68 & $2 a$ & Obesity \\
\hline & 21 & $\mathbf{F}$ & 42 & 74 & $2 b$ & Heberden's nodes. Rheumatic heart disease \\
\hline & 22 & $\mathbf{F}$ & 41 & 49 & $2 b$ & Mother with rheumatoid arthritis \\
\hline & 23 & $\mathbf{M}$ & 50 & 62 & $2 b$ & Histology \\
\hline & 24 & $\mathbf{F}$ & 74 & 89 & $2 b$ & Gall stones. Hypothyroidism \\
\hline & 25 & $\mathbf{F}$ & 77 & 77 & $2 b$ & $\begin{array}{l}\text { Hypertension. Ischaemic heart disease } \\
\text { Nephrectomy for hydronephrosis at age } 57\end{array}$ \\
\hline & 26 & $\mathbf{F}$ & 30 & 78 & $2 \mathrm{~b}$ & Hypothyroidism. Cataracts. Heberden's nodes \\
\hline & 27 & $\mathrm{~F}$ & 63 & 68 & $2 \mathrm{~b}$ & Basal bronchiectasis \\
\hline \multirow{3}{*}{3} & 28 & $\mathrm{~F}$ & 42 & 62 & 3 & Histology \\
\hline & 29 & $\mathbf{F}$ & 34 & 74 & 3 & $\frac{1}{0}$ \\
\hline & 30 & $\mathbf{M}$ & 51 & 67 & 3 & Diabetes. Hypertension. Histology \\
\hline \multirow{3}{*}{4} & 31 & $\mathbf{M}$ & 35 & 59 & $4 a$ & Recurrent podagra \\
\hline & 32 & $\mathbf{M}$ & 57 & 60 & $4 \mathrm{a}$ & Pernicious anaemia. Brother and uncle with "gout" \\
\hline & 33 & $\mathrm{~F}$ & 54 & 58 & $4 \mathrm{~b}$ & $\omega$ \\
\hline \multirow{2}{*}{5} & 34 & $\mathbf{F}$ & 57 & 57 & $5 a$ & $\begin{array}{l}\text { Renal stones } \\
\text { Parathyroid adenoma resected at age } 55\end{array}$ \\
\hline & 35 & $\mathbf{F}$ & 77 & 79 & $5 b$ & $\begin{array}{l}\text { Hyperparathyroidism } \\
\text { Heberden's nodes }\end{array}$ \\
\hline
\end{tabular}


IDIOLOGICAL CALCIFICATION OF JOINT CARTILAGE

\begin{tabular}{|c|c|c|c|c|c|}
\hline $\begin{array}{c}\begin{array}{c}\text { Serum } \\
\text { Calcium }\end{array} \\
\text { (mg. per cent.) }\end{array}$ & $\begin{array}{c}\text { Serum } \\
\text { Inorganic } \\
\text { Phosphorus } \\
\text { (mg. per cent.) }\end{array}$ & $\begin{array}{c}\text { Alkaline } \\
\text { Phosphatase } \\
\text { (K.A. units) }\end{array}$ & $\begin{array}{c}\text { Plasma } \\
\text { Uric } \\
\text { Acid } \\
\text { (mg. per cent.) }\end{array}$ & $\begin{array}{l}\text { Blood } \\
\text { Urea } \\
\text { (mg. per cent.) }\end{array}$ & $\begin{array}{l}\text { Synovial Fluid } \\
\text { Crystals }\end{array}$ \\
\hline $10 \cdot 0$ & $2 \cdot 2$ & $12 \cdot 1$ & $3 \cdot 2-9 \cdot 0$ & 20 & Not examined \\
\hline $8 \cdot 5$ & $2 \cdot 6$ & $8 \cdot 6$ & $3 \cdot 8$ & 27 & Few small e/c. Type uncertain \\
\hline $9 \cdot 3-9 \cdot 9$ & $2 \cdot 4-3 \cdot 0$ & $11 \cdot 6-13 \cdot 8$ & $3 \cdot 8-5 \cdot 3$ & 23 & Few e/c urate \\
\hline $9 \cdot 7$ & $3 \cdot 2$ & $6 \cdot 5$ & $6 \cdot 2$ & 24 & Numerous $\mathrm{i} / \mathrm{c}+\mathrm{e} / \mathrm{c}$ CPP \\
\hline $9 \cdot 4$ & $3 \cdot 3$ & $13 \cdot 4-23 \cdot 0$ & $2 \cdot 0-7 \cdot 0$ & 58 & $\begin{array}{l}\text { (1) } \mathrm{i} / \mathrm{c} \mathrm{CPP}+\mathrm{e} / \mathrm{c} \mathrm{CaOx} \\
\text { (2) } \mathrm{i} / \mathrm{c}+\mathrm{e} / \mathrm{c} \mathrm{CaOx}\end{array}$ \\
\hline $9 \cdot 4$ & $3 \cdot 4$ & $12 \cdot 6-17 \cdot 7$ & $7 \cdot 2-7 \cdot 5$ & 37 & CPP (in synovial biopsy) \\
\hline $9 \cdot 5$ & $2 \cdot 4$ & $5 \cdot 9$ & $3 \cdot 5$ & 22 & Not examined \\
\hline $9 \cdot 2$ & $2 \cdot 7$ & $5 \cdot 5$ & $4 \cdot 4$ & 40 & Not examined \\
\hline $9 \cdot 5$ & $4 \cdot 2$ & $10 \cdot 8$ & $6 \cdot 9-6 \cdot 9$ & $54-64$ & Few e/c small. Type undetermined \\
\hline $8 \cdot 4$ & $3 \cdot 2$ & $8 \cdot 3-13 \cdot 1$ & $3 \cdot 2$ & 25 & Numerous $i / c+e / c$ CPP \\
\hline $10 \cdot 2$ & $3 \cdot 4$ & $4 \cdot 0$ & $2 \cdot 2$ & 30 & Numerous $\mathrm{i} / \mathrm{c}+\mathrm{e} / \mathrm{c} \mathrm{CPP}$ \\
\hline $9 \cdot 8$ & $3 \cdot 6$ & $9 \cdot 5$ & $5 \cdot 0-7 \cdot 8$ & 25 & Moderate i/c+e/c CPP \\
\hline $9 \cdot 5$ & $2 \cdot 3$ & $9 \cdot 5$ & $7 \cdot 4$ & 40 & Not examined \\
\hline $10 \cdot 4$ & $3 \cdot 7$ & $11 \cdot 2-12 \cdot 7$ & $5 \cdot 3$ & $67-59$ & Moderate i/c CPP \\
\hline $10 \cdot 1$ & $3 \cdot 4$ & $7 \cdot 6$ & $5 \cdot 9-6 \cdot 9$ & 43 & $\begin{array}{ll}\text { (1) } & \text { Nil } \\
\text { (2) } & \text { Few e/c CPP }\end{array}$ \\
\hline \multirow[t]{2}{*}{$9 \cdot 5$} & $2 \cdot 6$ & $14 \cdot 2-15 \cdot 7$ & $4 \cdot 0$ & 27 & Nil \\
\hline & & $22 \cdot 2-28$ & & 25 & Not examined \\
\hline $9 \cdot 9$ & $2 \cdot 4$ & $5 \cdot 9$ & $6 \cdot 5$ & 84 & Moderate $i / c+e / c$ CPP \\
\hline $9 \cdot 6$ & $2 \cdot 5$ & $14 \cdot 2$ & $3 \cdot 9-4 \cdot 4$ & 45 & Moderate e/c CPP + CaOx \\
\hline $9 \cdot 9$ & $1 \cdot 7$ & $11 \cdot 8$ & $4 \cdot 0$ & 42 & $\begin{array}{c}\text { Sparse e/c } \\
\text { identified }\end{array}$ \\
\hline $8 \cdot 5-9 \cdot 0$ & $3 \cdot 7$ & $9 \cdot 4$ & & 30 & Few e/c +i/c CPP \\
\hline $9 \cdot 8$ & $2 \cdot 5$ & $4 \cdot 2$ & $3 \cdot 4$ & 34 & Moderate i/c CPP \\
\hline $9 \cdot 0$ & $3 \cdot 7$ & $14 \cdot 0$ & $3 \cdot 9-4 \cdot 2$ & 44 & Few e/c CPP \\
\hline $9 \cdot 9$ & $2 \cdot 5$ & $9 \cdot 9$ & $5 \cdot 0$ & 46 & Not examined \\
\hline $9 \cdot 2$ & $2 \cdot 6$ & $7 \cdot 8$ & $5 \cdot 7$ & 62 & Not examined \\
\hline $8 \cdot 5$ & $3 \cdot 4$ & $8 \cdot 0$ & $3 \cdot 0-4 \cdot 4$ & 48 & Not examined \\
\hline $9 \cdot 8$ & $3 \cdot 1$ & $6 \cdot 6$ & $5 \cdot 2$ & 42 & Not examined \\
\hline $8 \cdot 7$ & $3 \cdot 3$ & $6 \cdot 0$ & $2 \cdot 6$ & 15 & $\begin{array}{l}\text { Mixture-mainly e/c } \\
\text { Some CaOx. Many not identified }\end{array}$ \\
\hline $9 \cdot 6$ & $2 \cdot 6$ & $9 \cdot 0$ & $5 \cdot 1$ & 40 & Not examined \\
\hline $10 \cdot 0$ & $4 \cdot 0$ & $9 \cdot 0$ & $4 \cdot 0-5 \cdot 2$ & 38 & Few small $i / c+e / c$ Type uncertain \\
\hline $10 \cdot 8$ & $4 \cdot 1$ & $6 \cdot 2$ & $9 \cdot 0$ & 38 & Numerous $\mathrm{i} / \mathrm{c}$ and $\mathrm{e} / \mathrm{c}$ urate \\
\hline $10 \cdot 7$ & $3 \cdot 6$ & $9 \cdot 3$ & $4 \cdot 8-8 \cdot 6$ & 30 & Not examined \\
\hline $9 \cdot 6$ & $2 \cdot 6$ & $9 \cdot 0$ & $7 \cdot 3-13 \cdot 5$ & 30 & $\mathrm{i} / \mathrm{c}+\mathrm{e} / \mathrm{c}$ urate $\mathrm{e} / \mathrm{c} \mathrm{CaOx}$ \\
\hline $12 \cdot 6^{*} / 10 \cdot 3$ & $2 \cdot 0^{*} / 4 \cdot 1$ & $7 \cdot 8^{*} / 7 \cdot 3$ & * 13.6 & $27^{*} / 45$ & Not examined \\
\hline $10 \cdot 1-11 \cdot 2$ & $2 \cdot 6 \cdot 3 \cdot 5$ & $15 \cdot 4-18 \cdot 8$ & $3 \cdot 9-5 \cdot 6$ & 40 & $\begin{array}{l}\text { (1) Moderate } i / c+e / c \mathrm{CaOx} \\
\text { (2) Moderate } \mathrm{i} / \mathrm{c}+\mathrm{e} / \mathrm{c} \mathrm{CaOx}\end{array}$ \\
\hline \multicolumn{6}{|c|}{$\begin{array}{l}\mathrm{e} / \mathrm{c}=\text { extracellular } \\
\mathrm{i} / \mathrm{c}=\text { intracellular } \\
\text { Where more than two estimations have been carried out, only the highest and lowest readings are given. }\end{array}$} \\
\hline
\end{tabular}




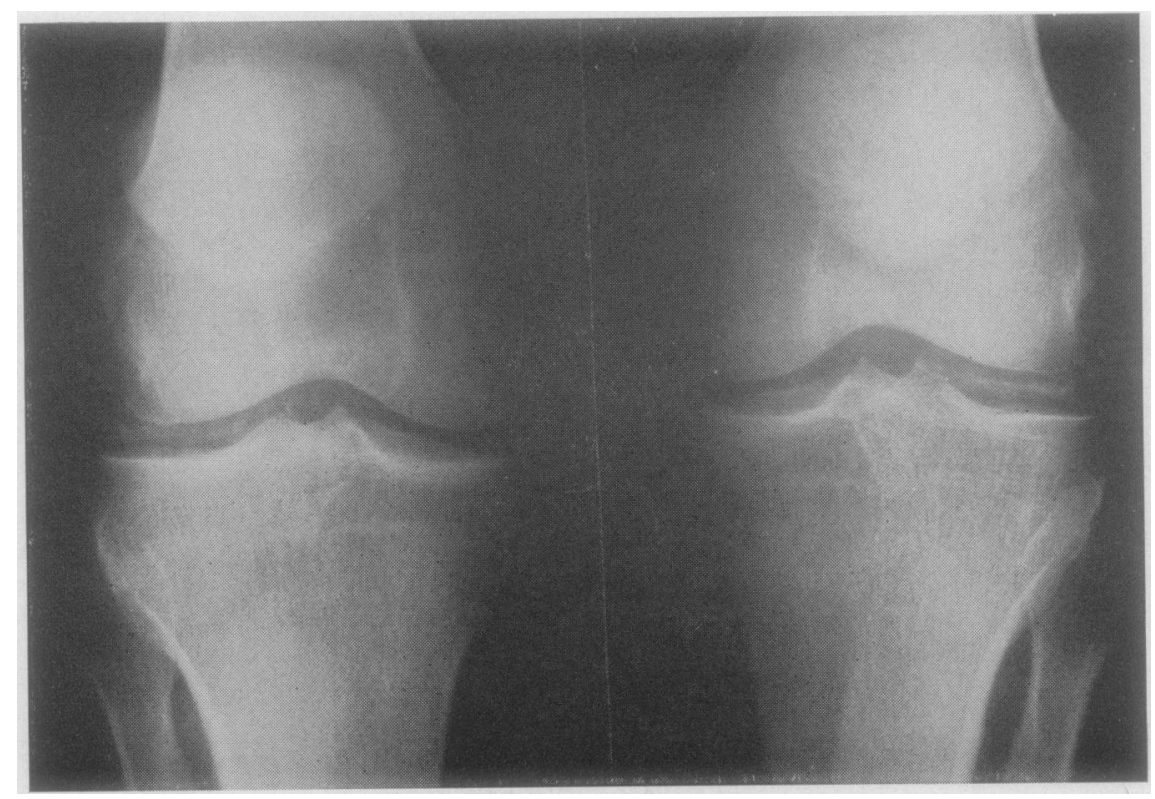

Fig. 1.-Case 4, radiograph of both knees. Calcification is present in the menisci of the knee, more marked in both lateral menisci. Fine broken lines of calcification are present close to the lateral

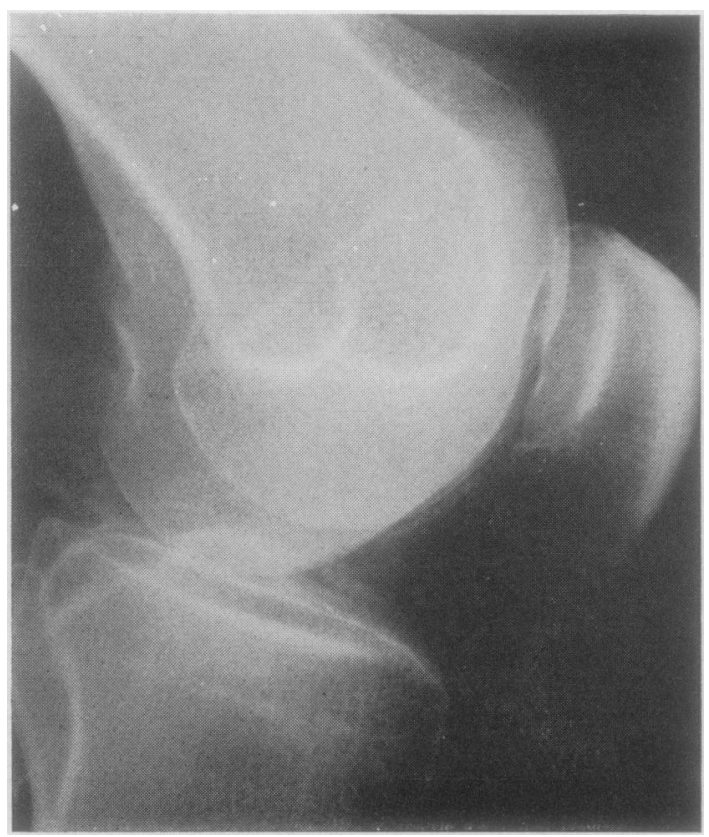

Fig. 2.-Case 4, lateral radiograph of the left knee. A line of hyaline calcification parallel to the femoral condyle is seen in addition to the meniscal calcification.

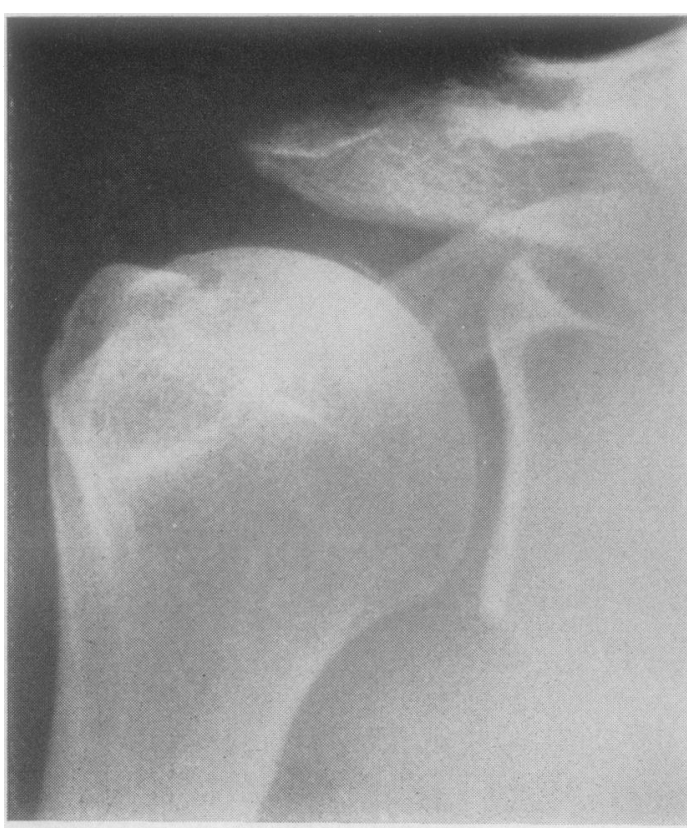

Fig. 3.-Case 4, radiograph of the right shoulder. A line of calcification parallel to the humeral head lies in the region of the articular cartilage. 


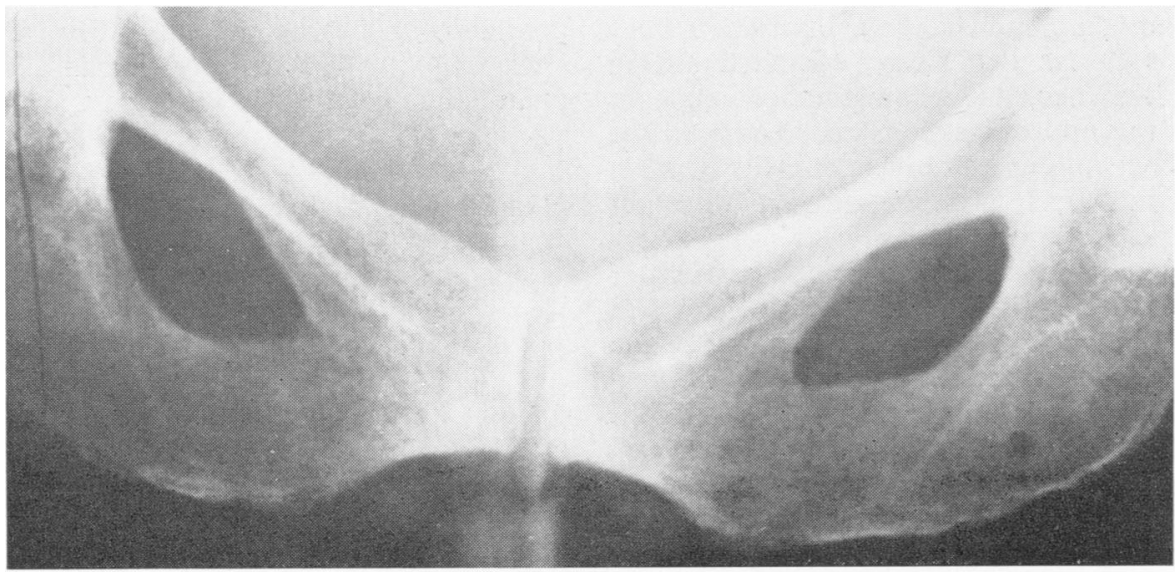

Fig. 4.-Case 9, radiograph of symphysis pubis. A vertical line of calcification in the fibrocartilage of the symphysis pubis lies between the bodies of the pubic bones.

Fig. 5.-Case 34, radiograph of the right wrist. Calcification is seen in the region of the fibrocartilaginous triangular disc.

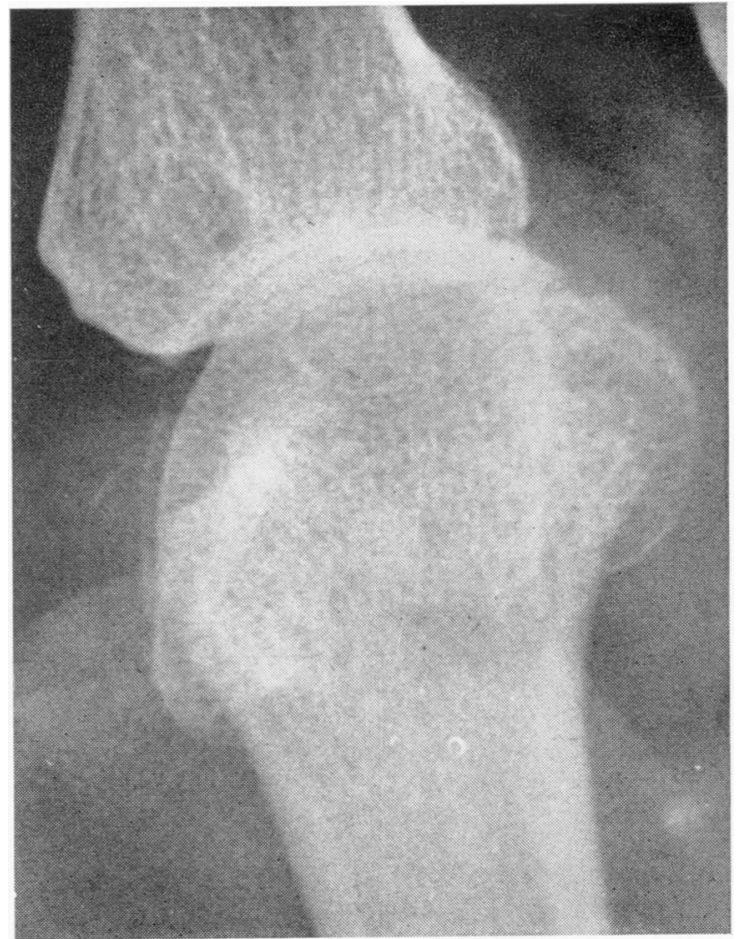

Fig. 6.-Case 30, detail of radiograph of right hand. A line of hyaline calcification parallels the head of the 2nd metacarpal and a little is seen near the base of its proximal phalanx. 
negative birefringence. Five fluids contained crystals which were diamond-shaped and varied from 3 to 6 microns in length. These exhibited strong positive birefringence, and the extinction angle in the long axis was approximately 30 degrees from the side face. They appear to be identical with crystals of calcium oxalate. However, as we have not yet succeeded in measuring exactly the refractive indices, nor in obtaining $x$ ray diffraction patterns of this material, the identification is tentative. We were interested on two occasions to find these crystals in the synovial fluid of a patient with hyperparathyroidism (Case 35). Four fluids contained crystals of various other forms, usually small, and either isotropic or positively birefringent, which we were not able to identify. Four fluids contained more than one type of crystal. The synovial fluid findings in each patient are indicated in Table III.

Crystals were often very sparse, but in only two specimens were they completely absent. During the period of this study, routine examination of fluids from patients without joint calcification has occasionally also revealed the presence of crystals. Usually these have been small and sparse, and difficult to identify, but we are satisfied that we have occasionally seen crystals of calcium pyrophosphate, urate, and the crystals referred to above, which may be calcium oxalate, in patients without calcification whom we regard as suffering from rheumatoid, psoriatic, or other inflammatory arthropathies. One patient with classical gout had a knee effusion containing crystals of both urate and calcium pyrophosphate.

\section{Histology}

Histological material was available from five patients. In Cases 6 and 17 synovial biopsies had been carried out earlier, and we were able to review the sections.

Case 6 (synovium from shoulder joint).-This had previously been reported as showing non-specific changes. On review the synovium was seen to be very vascular and contained a few multinucleate giant cells surrounded by round cells. Under crossed polars the giant cells were seen to contain crystals of calcium pyrophosphate (Fig. 7).

Case 17 (synovium from knee joint).-This section was also very vascular, with evidence of haemorrhage. Small groups of lymphocytes and plasma cells were present, but neither giant cells nor crystals were seen. These changes were regarded as non-specific, but the possibility that this joint was the site of early rheumatoid changes cannot be excluded. During the subsequent 2 years she has developed no rheumatoid phenomena. although the latex test was positive $(1: 40)$ on one occasion.

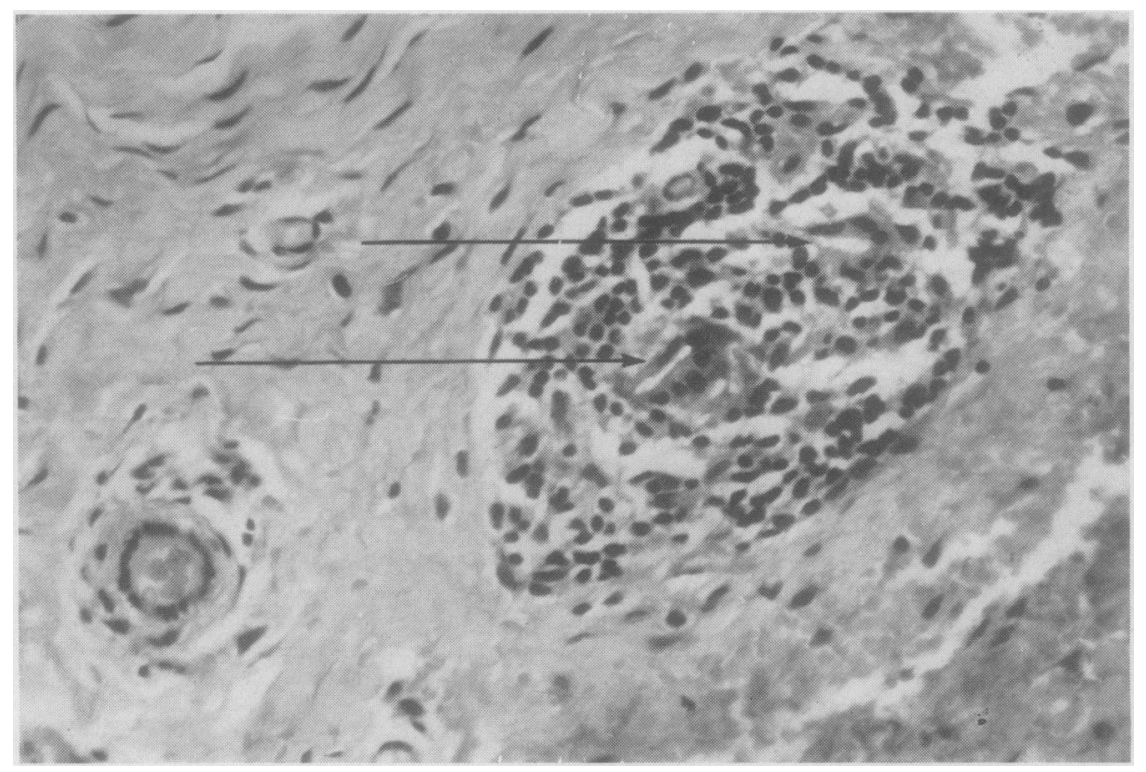

Fig. 7.-Cas -6 , synovium from shoulder joint. Arrows indicate crystals of calcium pyrophosphate lying within multinucleate giant cells. Partially-crossed polars produce birefringent effect. $\times 500$. 
Cases 23, 28, and 30.-These three patients each had an orthopaedic procedure carried out on a knee or hip joint, the site of advanced osteo-arthrosis. At operation areas were seen where the remaining joint cartilage was covered with a white "powdering" of crystalline material. Microscopically the synovia were hyperaemic and showed, again, non-specific changes. The cartilage was markedly disorganized in all three cases, and it is not possible to comment on the manner in which the crystalline material was laid down. Fig. 8 shows a disorganized meniscus impregnated with birefringent material.

Crystalline material was present in the histological material from all three of these cases. It appeared to be a mixture of calcium pyrophosphate and clumps of smaller, negatively birefringent, crystals, resembling apatite.

\section{Discussion}

These results indicate that calcification of joint cartilage is an unexpectedly common finding and, in this series, always associated with joint disease. Our cases were selected, in that each had been referred for radiography of the joint, but we find ourselves increasingly impressed with the significance of this sign in the investigation of arthropathy. Having started the study believing that calcification of a knee meniscus is often an isolated and unimportant finding, we have found that fuller investigation usually reveals calcification and arthropathy in other sites. The distribution of calcification within each joint, and the pattern of joint calcification generally, is strikingly stereotyped from patient to patient, which suggests that it is a feature of a generalized disorder, and the cause of the arthropathy, rather than the result.

Recent authors (McCarty and Gatter, 1964; Bundens, Brighton, and Weitzman, 1965) suggest that, whatever may cause the calcification, the associated arthropathy results directly from its presence; the calcification either produces cartilage damage locally, leading to osteo-arthrosis, or crystals enter the joint cavity to provoke a synovitis. To some extent our clinical material fits this hypothesis. Our two largest diagnostic groups ( 1 and 2) might be considered examples of the crystal synovitis and degenerative mechanisms respectively. But there are difficulties. In the crystal synovitis group, examination for crystals has given us far from clear-cut information. In three cases, calcium pyrophosphate crystals were certainly present in considerable numbers during an acute attack, but in others (not all examined during acute attacks) crystals were sparse and were not always identified as calcium pyrophosphate (see Table II). Examination for crystals did not clearly separate these cases from Group 2 patients (presenting as osteo-arthrosis), nor from the occasional patient with other forms of arthropathy (without calcification) whose joint

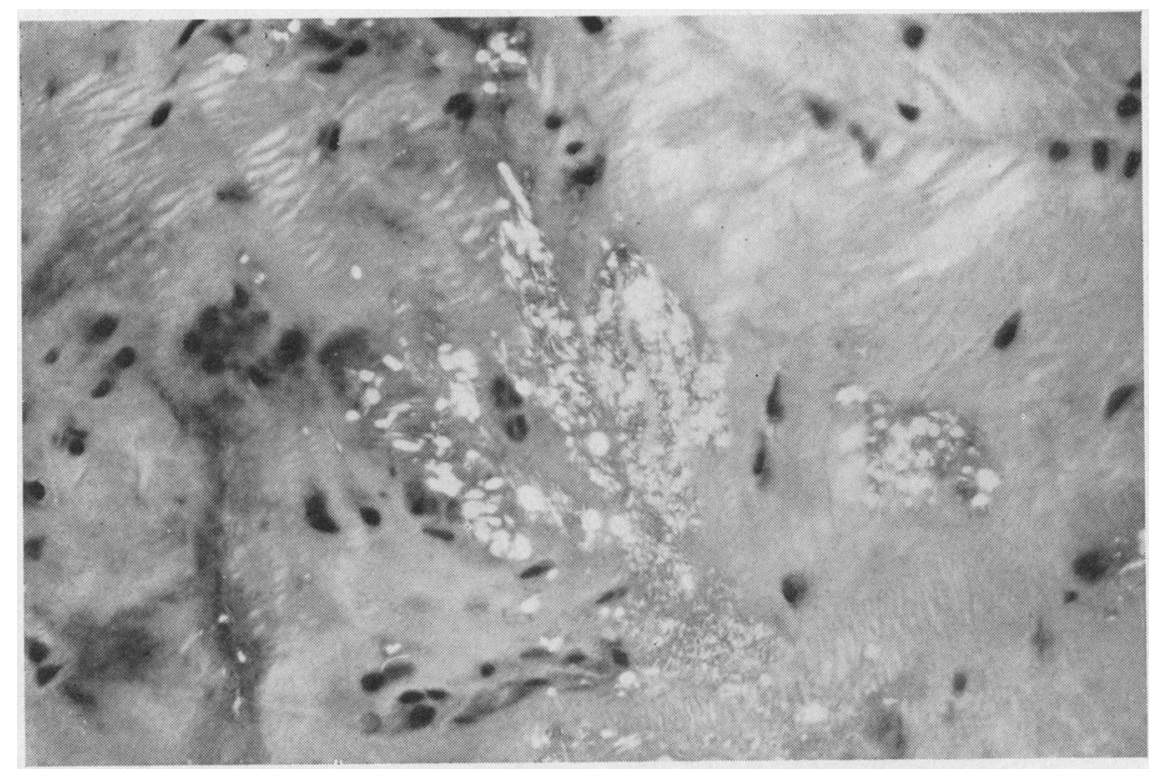

Fig. 8.-Case 23, knee meniscus. Disorganized fibrocartilage impregnated with crystals showing birefringence under partiallycrossed polars. $\times 500$. 
fluid contained crystals. This is in marked contrast to the clear-cut result that we have come to expect from polariscopic examination of fluids from subjects with classical gout.

The patients who presented with degenerative joint disease came from an age group in whom osteo-arthrosis is common, and any estimate of the part played by chondrocalcinosis has to take this into account. Osteo-arthrosis due to crystal deposition might be expected to affect mainly joints showing radiological calcification. This is so in a number of our patients, in whom a few large weight-bearing joints are the site of both calcification and degeneration. However, four of this group showed a pattern of "primary generalized osteoarthritis" (Kellgren and Moore, 1952) with marked degeneration in joints never seen to calcify (distal interphalangeal and first carpometacarpal) and complete freedom from arthrosis in the wrist, a very common site for calcification. While it is possible that lesser degrees of calcification, inapparent radiologically, may be a much more common cause of degenerative joint disease than is at present appreciated, this lack of congruency between the distribution of calcification and degenerative changes would seem to make this unlikely in what we prefer to call "nodal multiple osteo-arthrosis".

Of interest was the occurrence in three patients of a symmetrical polyarthritis mimicking rheumatoid arthritis. All three had been diagnosed as suffering from rheumatoid arthritis, but careful investigation showed no evidence of this. Synovial biopsies in two showed no rheumatoid features, and examination of synovial fluid obtained at operation was not helpful. A fourth patient showing a similar picture, with symmetrical small and large joint polyarthritis and erythrocyte sedimentation rate elevated to $130 \mathrm{~mm}$., we regarded as suffering from uric acid gout. She was consistently hyperuricaemic, her joint fluid contained mainly urate crystals, and the condition responded to colchicine. It seems likely that this last patient had urate crystal synovitis simultaneously in many joints. By analogy, calcium pyrophosphate crystal synovitis may have been the mechanism in the first three patients.

The primary defect leading to joint calcification remains unexplained. We are impressed by the frequency with which cartilage calcification is associated with soft tissue calcification in periarticular structures, blood vessels, and other sites. This suggests a general metabolic defect rather than defective cartilage. Unexpectedly, we have not found any increase in costal cartilage calcification.

The radiological pattern of joint calcification is $\overrightarrow{\vec{S}}$ strikingly similar from patient to patient, and be- $\overline{0}$ tween the various published series. This is in con- 흘 trast to the marked genetic and metabolic differences between the groups showing this common $\mathbb{\Phi}$ radiological sign. Any hypothesis about the primary defect must take into account these differences. Žitňan and Sitaj (1963) described severe? involvement in relatively young subjects with no $\vec{\omega}$ demonstrable metabolic abnormality, who came o from one locality and showed a marked familial $\frac{\varrho}{2}$ aggregation. Our material is similar to that of McCarty and Gatter (1964). Like these authors or and others, we have found that, while most of our patients show no gross metabolic abnormality, an o appreciable minority have convincing evidence of 0 either hyperparathyroidism or classical gout. Between these two extremes are a number of patients with either unexplained hyperuricaemia or raised levels of serum alkaline phosphatase. There may $\overrightarrow{0}$ be an increased incidence of diabetes in patients with chondrocalcinosis and there is certainly an association with haemochromatosis (Serre, Simon, ando Sany, 1965; de Sèze, Solnica, Habault, Jaffres, Mitrovic, and Kahn, 1965; Jaffres, 1965).

The incidence of joint calcification in unselected $\stackrel{\mathbb{\Omega}}{\rightarrow}$ subjects is unknown. Bocher, Mankin, Berk, andôㄹ Rodnan (1965) found no less than 10 per cent. of $100^{3}$ elderly subjects (average 80 years) to have calcified menisci. Lawrence (1965) found an incidence of five out of 1,619 in a population survey of persons over 34 years of age. Amongst our patients there was no tendency for calcification to be more marked 3 . in older subjects.

The role of inorganic pyrophosphate compounds in intermediate metabolism is not known. Recents work suggests that they may be a "normal" substrate. of alkaline phosphatase and may play a part in then control of bone mineralization by inhibiting crystaP growth (Fleish and Neuman, 1961; Russell, 1965) N Further work along these lines may well throw light on the mechanism of articular cartilage calcificationg 
This was equally true whether the calcification affected many joints or only one.

Patients showing this radiological sign fell into one of three clinical categories:

(1) Episodic acute arthritis of one or a few large joints (some going on to develop degenerative changes).

(2) Degenerative joint disease (some having acute exacerbations).

(3) Small joint polyarthritis mimicking rheumatoid arthritis.

Most patients showed no evidence of a metabolic abnormality, a few had either frank gout or hyperparathyroidism. Others had either hyperuricaemia or raised levels of serum alkaline phosphatase.

In the majority of these patients it is felt that the crystal deposition was the cause of the arthropathyeither by causing local degeneration of cartilage or by entering the joint to provoke a crystal synovitis. In a few patients joint cartilage calcification was thought to be coincidental with other forms of arthropathy-nodal multiple osteo-arthrosis and uric acid gout (cf. Moskowitz and Katz, 1965).

Examination of synovial fluids for crystals proved a disappointing method of categorizing these patients. In three instances numerous crystals of calcium pyrophosphate were found in fluid from acutely inflamed joints. Most other specimens contained crystals, usually sparse, and consisting of either calcium pyrophosphate or other compoundssome unidentified. Our experience suggests that this investigation (so valuable in uric acid gout) should be interpreted as indicating calcium pyrophosphate crystal synovitis only when crystals are numerous.

The cause of joint cartilage calcification is unknown. A general metabolic defect appears more likely than a primary lesion of cartilage. A number of different mechanisms may be capable of producing the same radiological appearance.

This subject is becoming overburdened with names. Only two appear worth retaining: chondrocalcinosis articularis (for describing a radiological sign) and crystal synovitis.

\section{Summary}

In order to evaluate the significance of radiological calcification of joint cartilage, 35 consecutive cases $\overrightarrow{\vec{c}}$ showing calcification in the knee were investigated.

All cases showed evidence of arthropathy. In the $\frac{\overline{\bar{O}}}{\bar{D}}$ majority, this was thought to be secondary to the $\frac{\vec{\sigma}}{\sigma}$ calcification. A minority of cases showed evidence $\varrho$ of some metabolic abnormality. The clinical \& features and laboratory investigations (including the $\vec{\circ}$ examination of joint fluids for crystals) are described and their significance discussed.

We are grateful to our colleagues on the staff of the London Hospital and to Dr. J. H. Glyn and Dr. G. E. N Storey for allowing us to study patients under their care. The photomicrographs were kindly prepared by $\mathrm{Dr}$. त C. L. Brown.

\section{REFERENCES}

Bocher, J., Mankin, H. J., Berk, R. N., and Rodnan, G. P.气 (1965). New Engl. J. Med., 272, 1093.

Brit. med. J. (1964). Leading Article, 2, 889.

Bundens, W. D. (Jr.), Brighton, C. T., and Weitzman, G. (1965). J. Bone Jt Surg., 47-A, 111.

Bywaters, E. G., Dixon,"A. St.J., and Scott, J. T. (1963). Ann. rheum. Dis., 22, 171.

Fleish, H., and Neuman, W. F. (1961). Amer. J. Physiol.,,$\stackrel{2}{\mathbb{Q}}$ 200, 1296.

Hamblen, D. L., Currey, H. L. F., and Key, J. J. (1966). J. Bone Jt Surg., 48-B, 51.

Jaffres, R. (1965). Ouest méd., 18, 751.

Kellgren, J. H., and Moore, R. (1952). Brit. med. J., 1, 181.

Key, J. J., and Currey, H. L. F. (in press).

Lawrence, J. S. (1965). Personal communication.

McCarty, D. J., Jr., and Gatter, R. A. (1964). Bull. rheum. Dis., 14, 331.

Kohn, N. N., and Faires, J. S. (1962). Ann. intern. Med., 56, 711.

Moskowitz, R. W., and Katz, D. (1965). Arch. intern. Med., 115, 680.

Russell, R. G. G. (1965). Lancet, 2, 461.

Serre, H., Simon, L., and Sany, J. (1965). Congr. Rheum. Mar del Plata, Argentina.

Sèze, S. de, Solnica, J., Habault, R., Jaffres, D., Mitroviao D., and Kahn, M. F. (1965). Idem.

Žitñan, D., and Sit'aj, S. (1958). Bratisl. lek. Listy, 28, 217.

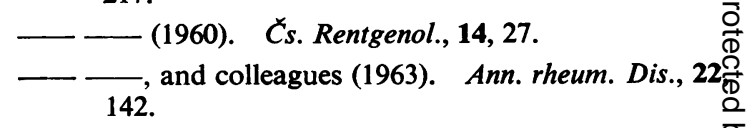


L'importance de la calcification radiologique du cartilage articulaire

RÉSUMÉ

Afin d'évaluer l'importance de la calcification radiologique du cartilage articulaire, on a examiné 35 cas consécutifs de calcification au genou.

Dans tous les cas on a trouvé des signes d'arthropathie. Dans la plupart de ces cas on l'a considéré secondaire à la calcification. Dans la minorité des cas on a trouvé des signes d'une anomalie métabolique. On décrit les caractères cliniques et de laboratoire (y compris la recherche de cristaux dans le liquide articulaire) et on discute leur importance.
La significación de la calcificación radiológica del cartílago articular

\section{SUMARIO}

Para valorar la significación de la calcificación radiológica del cartílago articular, se examinaron 35 casos consecutivos de calcificación en la rodilla.

En todos los casos se encontró evidencia de artropatía. En la mayoría de estos casos esta artropatía fué considerada secundaria a la calcificación. En una minoría de ellos se vieron signos de anomalía metabólica. Se describen los es rasgos clínicos y de laboratorio (incluso con la busca de $\overrightarrow{0}$ cristales en el líquido articular) y se discute su importancia. 